\title{
Design of The Data Acquisition System Based on STM32
}

\author{
Pei-Xue Liu, ${ }^{1, a}$, Yu-jie Chen ${ }^{1, b}$, Bao-Hua Jiang ${ }^{1, c}$ and Xiao Zhang ${ }^{1, d}$ \\ ${ }^{1}$ Qingdao HuangHai College, Shandong, China \\ a93987398@qq.com, b79572611@qq.com, c1073578901@qq.com, ${ }^{\mathrm{d}} 1711301193 @ q q . c o m$, \\ *Pei-Xue LIU
}

Keyword: Stm32f103zet6; Temperature and humidity; GSM;

\begin{abstract}
With rapid development of modern society, the requirements for high precise temperature and humidity is growing rapidly, for example production, storage, transportation, environment etc., these all have strict requirements for temperature and humidity testing, so the real-time monitoring of temperature and humidity and alarm prompt is extremely important. In this paper, a temperature and humidity automatic alarm recorder is designed, which uses stm 32 as control center, combines the technique of sensor technology, GSM technology and serial port to finish button control measurement of temperature and humidity or two road all the way. In the study, the system's hardware and software design are introduced in detail and testing results show that the system can realize the function of the temperature and humidity measurement record and alarm.
\end{abstract}

\section{Introduction}

Along with the rapid development of modern society, people are increasingly demanding on the quality of life, production and living environment requirements need to be more and more detailed to meet the demand of people, some storage units such as petroleum, chemical, aerospace, pharmaceutical, food and other requirement for temperature and humidity is higher, so it is meaningful to have temperature and humidity of the research.

In this paper, we design a data acquisition system based on STM32, data can be sent via a serial port to the server. System not only can complete all the temperature and humidity data acquisition or two road temperature data collection, and can be anywhere at any time to the acquisition of the temperature and humidity data monitoring, once more than limit the buzzer alarm and send prompt message to the mobile terminal. The high efficiency than the traditional system, the resources use fully, and short measuring cycle, low cost, the staff don't have to site operation. Emerging technologies used in this system, has the scientific nature can avoid the happening of the accident, and the system has simple structure, high reliability, low cost, so the system has very important application value of the market.

\section{Overall design of system}

The temperature and humidity automatic alarm recorder is mainly composed of: data collection module, display module, alarm module, GPRS module, serial transmission module, memory module, the charging module of seven parts, such as the system overall design is shown in figure 1 .

Charging modules to the control center and each module power supply, control center control key choice of temperature and humidity of the acquisition or two road all the way temperatureacquisition, collect data and feedback to the control center for the operation of the alarm circuit and display module, once reach the limit temperature and humidity, the control center control SIM900A 


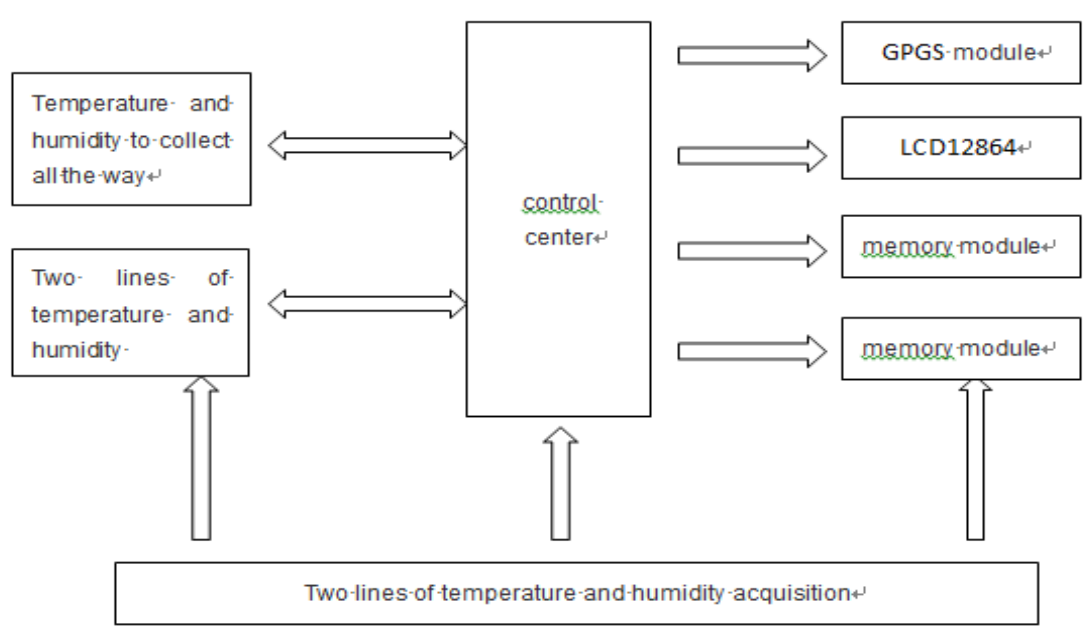

Figure 1 overall system design

module will alarm prompt messages sent to the mobile terminal and start the buzzer, data collection terminal through a serial port sent to the server and start the control module used AT24C02 modules store data.

\section{Hardware design}

\section{Temperature measurement circuit design}

18 b20 temperature sensor is able to output the digital temperature sensor, it is better than the traditional temperature sensor hardware, low cost, good stability, simple circuit connection and easy control. First DS18B20 is negative properly connected power supply and ground, DQ and control center MCU PD11 JiaoXiang connection for data transmission and control, because the sensor itself cannot output high level, so the data pins DQ external pull-up resistors R5 make SCM can read "1" signals, two temperature sensors are the same connection, the second DQ PC11 pin of MCU, to realize the collection of two way temperature temperature sensor DS18B20 connection circuit diagram (see figure 2).

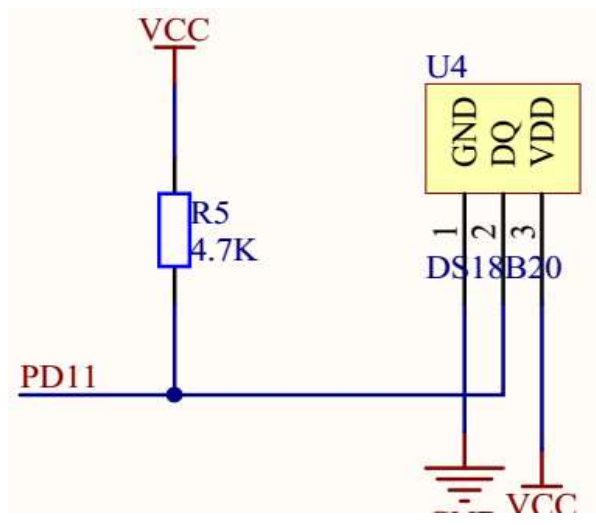

Figure 2 temperature measurement hardware circuit diagram

\section{The humidity measuring circuit design}

DHT11 is a single wire system serial mouth of temperature and humidity sensor, it combines temperature and humidity sensor technology and dedicated digital module acquisition technology as a whole, can directly output digital signal, its stability and reliability are high. DHT11 four pins, feet dangling $\mathrm{NC}$ is not connected to any device, two power supply pins to connect power and ground respectively, and the data pin MCU PG11 pin, control the sensor data acquisition and transmission, but the connection to connect a $5 \mathrm{k}$ on resistance, prevent microcontroller pins for low 
electricity at ordinary times, the external circuit can provide current to the pins. Temperature and humidity hardware connection diagram (see figure 3 ).

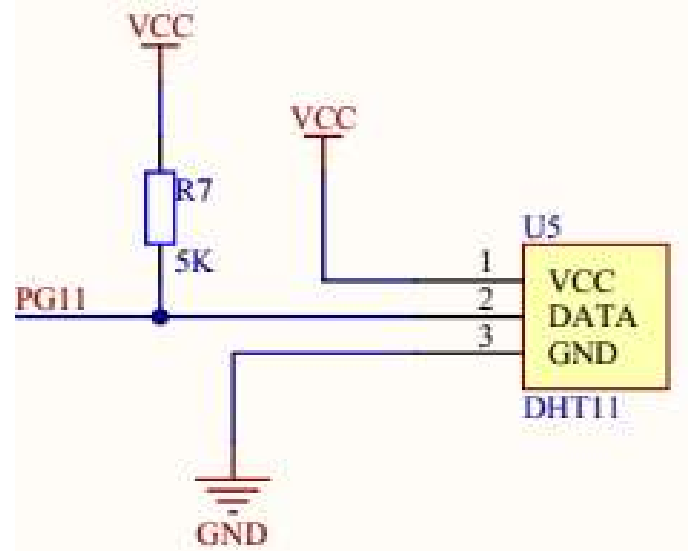

Figure 3 temperature and humidity measurement hardware circuit diagram

\section{The design of display circuit}

LCD12864 is a LCD screen can display the simplified Chinese character and general graphics, the interface simple connection, software control is also very convenient, 12864 LCD module 20 pins, there are two NC is empty feet is not connect any devices, and VD foot is equivalent to impending, R11 connected it with the slide rheostat with LEDA and LEDK control the display backlight degrees, 1 foot and 2 feet respectively connect the power supply and to provide the power for the whole module, DB0 DB7 12864 connecting MCU PC0 - PC7 control LCD display content, RS, RW, EN, RSB, RES respectively connected to the microcontroller PG13, PG14, PG15, PB3, PG4, by controlling the high and low level of each pin [16], complete LCD screen display, the port connection (see figure 4).

\section{The software design and test}

\section{software design}

Boot after the initial configuration for equipment first and then choose between temperature and humidity measure or measurement of the temperature of two way all the way, choose the way to measure after enter into the state of the data collection, gathering the one sent to the control center, has been cycle collection. Device is powered on, first on the buttons and minimum system initialization, and then choose between temperature and humidity device initialization fortemperature and humidityafter sampling is complete without continues to collect or output to the control center (key)choice after temperature device initialization temperature sampling is complete

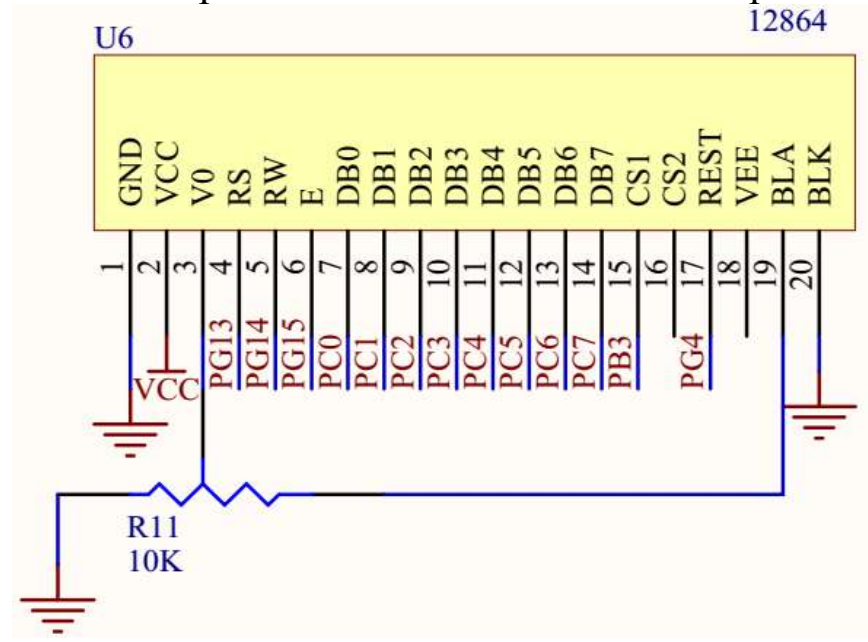

Figure 4 LCD display hardware circuit diagram 
without continued to acquisition or output data to control center). Acquisition program flow chart (see figure 5).

\section{system testing}

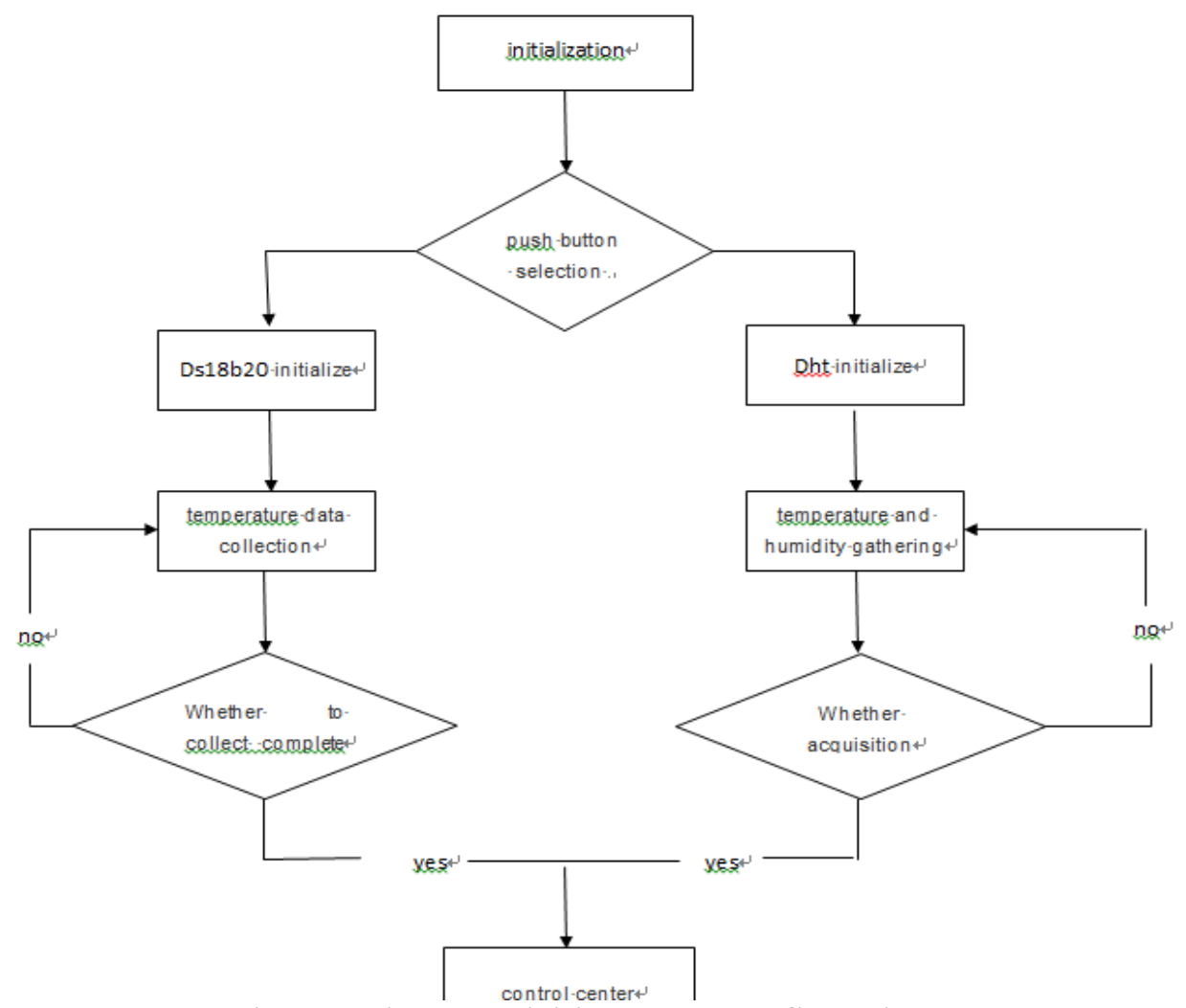

Figure 5 data acquisition program flow chart

This design is mainly composed of STM32F103ZET6 control the running of the whole system and send prompt SMS with GSM technology, through a serial port for data transmission, this test plan is to use work to make the prototype test, the design prototype (see figure 6).

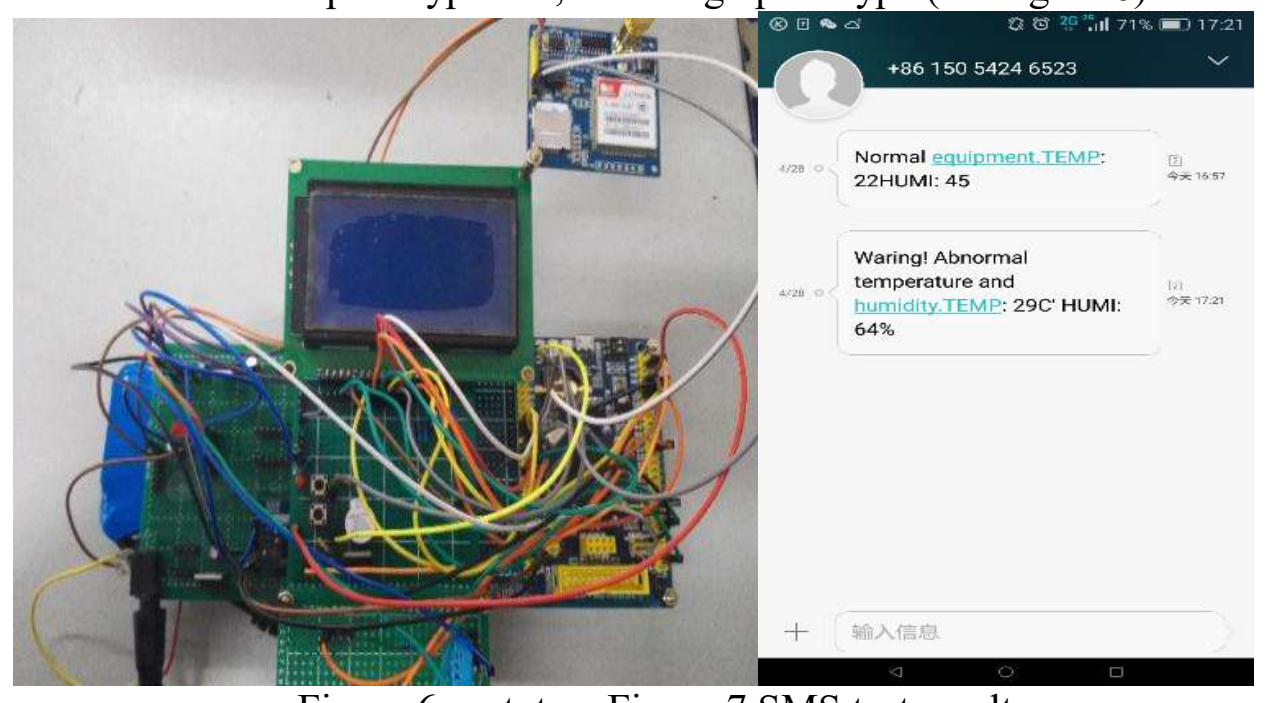

Figure 6 prototypeFigure 7 SMS test results

Whole running, this design is mainly controlled by the STM32f103ZET6 equipment run time by the first SIM900A module testing network connection, confirm that the network system run normally, can choose all the way through the button of the temperature and humidity acquisition or two road temperature gathering, gathering feedback data provide the basis for the control center, control LCD display measurements, and determine whether the temperature and humidity reaches 
limit, if do not conform to the requirements, the buzzer alarm and sent via GPRS module to the user mobile phone terminal business messages (as shown in figure 7).

\section{Summary}

The purpose of this design to design temperature and humidity automatic alarm recorder under a machine, the system hardware to complete function: can button control temperature and humidity temperature collection or two road, all the way to collect data on the LCD screen display, alarm and send hint to the user's phone text messages, alarm limit value can be modified by software, can record a day's temperature and humidity measurement data and through a serial port and PC communication, which the time intervals of the recorded data can be change by the program, the above function through the system test run is good, has the very high application value.

\section{Acknowledgement}

This research was financially supported by the College Science and technology project of Shandong Province The Design of Internet of things remote identification and information collection system based on4G(J15LN59)and the design of cloud platformfor internet of thins environment based on $\mathrm{B} / \mathrm{S}$.

\section{References}

[1] zhoubing, Ma Yingqing, Cindy. Intelligent temperature and humidity control instrument [J]. Instrument technique and sensor, 2002, 11:52-53.

[2] yu-fen Yang, ben-huazhang. Composition and development of intelligent temperature and humidity monitoring system [J]. Journal of agricultural mechanization, 2006, 2:52-101.

[3] zhangwei, dong-fengwang. New greenhouse temperature recorder system design [J]. Mechanical and electrical product development and innovation, 2009, 5:20-31.

[4] Wang Fulu. Cold to remote monitoring system for greenhouse research [D]. The northeast agricultural university, 2011. 